\title{
Editorial: Dawning of a New Age for Longitudinal Cohort Data
}

\author{
Richard Suzman • Sarah Harper
}

Published online: 13 April 2013

(C) Springer Science+Business Media Dordrecht 2013

Population ageing is changing the world in fundamental ways. It is a global phenomenon with profound short- and long-term implications for both macroeconomics and the social wellbeing, and the health and long-term care needs of many nations. However the context and timing of population ageing vary across and within world regions and countries. In particular the industrialized nations became wealthy before they aged significantly, while many of the poorer regions are ageing before they have become significantly industrialized. Meeting the variety of challenges will require significant advance planning and preparation. As longevity increases so will needs of economic security in old age as well as health and long term care. Extending working lives is among the few solutions to this macro problem, beyond increasing taxes, reducing consumption to save more for retirement, or increasing worker productivity. A key nationally representative study in the USA, the National Long Term Care Survey, showed, contrary to informed opinion, that ageing was plastic in that disability in the older population declined by as much as $25 \%$ between 1984-1999. Unfortunately, this very positive trend may not have continued., The long term care costs of Alzheimer's Disease, one of the most costly diseases in terms of both economics and family wellbeing, will rise significantly along with the rapid growth of the Oldest Old segment of the older population. The extent to which research can find solutions that reduce physical and cognitive disability at older ages will prove a significant factor in how both high and low income countries cope with this fundamental transformation of populations and societies.

The National Institute of Aging (NIA) is one of the 27 Institutes and Centers of the US National Institutes of Health. It leads the federal government in conducting and supporting research on ageing and the health and well-being of older people. The

R. Suzman

Division of Behavioral and Social Research at the National Institute on Aging (NIA),

National Institutes of Health, Bethesda, USA

S. Harper $(\square)$

Oxford Institute of Population Ageing, Oxford, UK

e-mail: sarah.harper@ageing.ox.ac.uk 
Institute seeks to understand the nature of ageing and the ageing process, and diseases and conditions associated with growing older, in order to extend the healthy, active years of life. The role of the National Institute of Aging includes a mission to improve our understanding of the consequences of an ageing society and provide that information to inform intervention development and policy decisions; to understand how population ageing and changes in social, economic, and demographic characteristics of cohorts reaching old age affect health and well-being; and to understand how social and economic factors throughout the lifespan affect health and well-being at older ages. As part of that mission, NIA has supported and facilitated many of the Longitudinal Studies explored in this special edition of the Journal of Population Ageing.

The history of nationally representative longitudinal studies that focus on ageing is quite recent, at least in the US. From 1969-1979 the Social Security Administration conducted the Retirement History Survey focusing on a narrow age segment. During the 1980s the NIA funded the National Long Term Care Study of Aging and the National Long term Care Survey. Indeed, all the studies in this edition were been established in the past two decades. The US Health and Retirement Study (HRS) was established to replace the old Retirement History Survey, but with a much greater emphasis on health as well as improved data collection on economic status. The was featured at the premeeting on population ageing that preceded the Denver Summit meeting of the G-7, and the G-7 Communiqué espoused the need for comparable data collection across multiple countries. The HRS model spread rapidly to other nations - including the English Longitudinal Study on Ageing (ELSA) and the Survey on Health and Retirement in Europe (SHARE). More or less comparable studies in Asia were developed in South Korea (KLOSA), China (CHARLS), Japan (JSTAR) and now India (LASI); for some such as Korea and Japan, the NIA and the its grantees provided only limited technical assistance, while for others the NIA provided substantial cofounding. Additionally in Europe studies was developed in Ireland (TILDA) and ones are being developed in Scotland and Northern Ireland. In the Americas, there is a Mexican study (MHAS) and one is being developed in Brazil. The NIA commissioned a series of regional reports on population ageing from the Committee of Population at the US National Academy of Science, and these reports were used to elicit interest in national data collection among various nations.

The studies varied in the level of data collected on health - some studies such as ELSA and TILDA developed more sophisticated data collection methods for health than the HRS - which in turn benefited and adopted some of their methods. These studies have become part of a web or network of researchers that have significantly increased the size of the international research community focusing on population ageing, and useful innovations in one site have often spread rapidly to studies in other countries. In addition the NIA funded the WHO to collect data on health and ageing in six countries (SAGE), including two African countries-Ghana and South Africa. A central motivation for NIA's funding of these national longitudinal study was to understand the evolution of disability and functioning, and to find out whether the decline in disability found using the National Long Term Care Survey, was also happening in other countries, and if so to discover common dynamics.

A key principle that has been followed by all of these studies is that the data are widely shared and the sharing occurs very rapidly after the data have been collected. 
Additionally there has been a concerted effort to harmonize the data collection and measures across the surveys to allow for more comparative analyses. Harmonization naturally elicits a tension between maintaining the history of measures already used — an important principle in longitudinal studies - and allowing for comparative analyses. Introducing cross-walked alternative measures can sometimes satisfy both demands. The NIA has provided support for harmonization and the RAND Survey Meta Data Repository maintains an efficient harmonized database for most of these studies.

Several comparative studies have had a significant impact. One study comparing the HRS and ELSA found that American health was significantly worse that English health even with SES and many risk factors controlled. Another study showed that England's health was generally inferior to the health of European countries, again with SES controlled. These studies resulted in a series of important reports from the US National Academy of Sciences and the Institute of Medicine on the inferiority of American health. Another comparative study using these studies discovered that working longer at older ages might be a factor in maintaining cognitive functioning.

A recent initiative is the collection of biological samples: blood and DNA, and two of the studies, the HRS and ELSA, have already conducted Genome Wide Association Studies (GWAS), and discussions have started about moving on to full sequencing. Genetic studies often require very large samples and this fact is another driving factor in the move to increase harmonization of various outcomes and measures. Integrating genetic information with environments and behavioural measures is an evolving one, and gradually this is leading to the development of behavioural measures that are more tightly anchored to biological pathways. Quite how the introduction of genome-wide genetic data into social and behavioural models will change the models remains to be seen, but the results might well be revolutionary, though some sceptics argue differently.

The NIA funds several other national longitudinal surveys with different goals from the HRS such as MIDUS, NHATS and NSHAP along with regional studies such as the Wisconsin Longitudinal Study. We are exploring options for harmonizing outcome measures such as cognition and momentary or experienced subjective wellbeing. In encouraging harmonization it is important at the same time to avoid homogenization of measures. We hope that this process might extend to other cohort studies including some featured in this special issue.

The sustainability and longevity of these studies is highly dependent on funding including government support. While the NIA has provided co-funding for several, but not all, of the studies in Asia and Europe, most studies have relied on their own national government bodies for support. However, during the current economic crisis, several countries, especially those that are part of SHARE, have encountered funding crises. Maintaining the funding for these studies will become ever more important as the impact of population ageing accelerates and as the value of the accumulated longitudinal data grows.

There is accumulating evidence from analyses of these databases of the crucial importance of early childhood health for health in adulthood and old age. Indeed one study of medical students and physicians in a US medical school found that despite having attained high social status as physicians, early childhood disadvantage still had a substantial impact on mortality in adulthood. Few countries have the benefit of the UK's 1946 and 1958 birth cohorts, and it has become necessary to develop methods to obtain valid and reliable evidence about childhood experiences from 50 or 80 year olds. 
One possibility is to use data from the few birth cohorts that have reached middle and old age to experiment with approaches that can best capture early childhood experiences. There are limits to what can be obtained by self report and another option is to increase the use of linked administrative data on education, health and income.

Some life scientists argue that observational data cannot be used to develop reliable and valid findings in many areas of health because of the considerable degree of confounding, even with controls. Some of these views arise from the failure in randomized trials of variables (or substances such as Vitamin E or beta carotene) suggested by epidemiological observational studies. These researchers argue that randomized controlled trials (RCTs) are the best method for developing high quality findings. This is too long and complicated an issue to treat fully in this editorial, but each method has advantages, disadvantages, and limitations. It is possible that combining both approaches may have substantial advantages. Some findings from complicated analyses of longitudinal data might increase in value if the findings can be assessed in experiments, including effect sizes. One paper in this special issue discusses the impact of living in disadvantaged housing and areas. One study cofunded by the NIA made use of random assignment of housing vouchers that allowed families to move to different types of housing with various degrees of advantage, and the indeed moving to better quality areas did improve several outcomes including measures of wellbeing. Another ongoing RCT is investigating the impact on low income elderly acting as volunteers in schools. The HRS made use of a natural experiment when subsidized prescription drugs were provided to the elderly for the first time. Another study made use of a lottery in the state of Oregon to observe the impact on health of providing health care insurance to the low income population (there were insufficient funds to provide the insurance to all who qualified). It is probable that in the future we will see closer integration of longitudinal observational studies and randomized experiments.

The underlying commonality in this collection is the existence of the mostly new longitudinal cohort data that are providing new opportunities to understand the dynamics of ageing. Important themes that emerge are the long reach of childhood into old age, the course of active life expectancy, cross national comparisons, harmonization of data sets, the importance of rapid sharing and archiving, linked administrative data, and the interplay between observational and natural experiments and randomized interventions.

This is surely one of the most exciting times for ageing research. As is clear from this edition, longitudinal life course studies around the world are providing a new and unique opportunity to explore key questions around ageing societies. 